\title{
Characteristics and clinical management of acute pulmonary embolism in real world: findings from TUSCAN-PE study
}

\author{
Luca Masotti, ${ }^{1,2}$ Filippo Pieralli, ${ }^{3}$ Grazia Panigada, ${ }^{4}$ Francesco Corradi, ${ }^{5}$ Rino Migliacci, ${ }^{6}$ Salvatore Lenti, ${ }^{7}$ \\ Alessandro Tafi, ${ }^{8}$ Carlo Nozzoli, ${ }^{3}$ Giancarlo Landini, ${ }^{2}$ for TUSCAN-PE Investigators \\ ${ }^{1}$ UO Medicina Interna, Ospedale di Cecina (LI); ${ }^{2}$ UO Medicina Interna, Ospedale Santa Maria Nuova, Firenze; \\ ${ }^{3}$ UO Medicina Interna 1, Ospedale Careggi, Firenze; ${ }^{4} \mathrm{UO}$ Medicina Interna, Ospedale di Pescia (PT); ${ }^{5} \mathrm{UO}$ Medicina Interna \\ 2, Ospedale Careggi, Firenze; ${ }^{6} \mathrm{UO}$ Medicina Interna, Ospedale di Cortona (AR); ${ }^{7} \mathrm{UO}$ Medicina Interna, Ospedale di Arezzo; \\ ${ }^{8}$ Tuscan FADOI President (2011-2013), Firenze, Italy
}

\begin{abstract}
Acute pulmonary embolism (PE) remains one of the leading causes of mortality and morbidity in cardiovascular setting. Despite much information about clinical aspects and recommendations or clinical guidelines is available from literature, few data exist about the management of PE in real world of internal medicine scenario. Therefore the aim of the present study was to report on characteristics and management of PE patients admitted in this setting. TUSCAN-PE study was a multicenter, observational, retrospective, cohort study aimed to analyze data of PE patients admitted in Internal Medicine wards of Tuscany. Each center was invited to submit anonymously data of at least ten patients consecutively discharged for acute PE in 2012. Data were referred to demographic, clinical, instrumental, prognostic and therapeutic characteristics. A total of 452 patients from 28 Tuscan centers $(60.2 \% \mathrm{~F})$, with mean age $76.01 \pm 12.34$ years, were enrolled. A total of $87 \%$ of patients was admitted from Emergency Department, but only $65.2 \%$ of patients with diagnosis of PE. Around one third of diagnoses of PE was performed by internists. In $14.8 \%$ of diagnoses was incidental. In $86 \%$ of patients, diagnosis was performed by computer tomography pulmonary angiography. Overall mortality was $9.5 \%, 5.75 \%$ being PE-related. Main risk factors enclosed recent respiratory tract infections (55.3\%), immobility (42.25\%), recent hospital admissions (33.6\%) and cancer (30.3\%). In 65.8\% of patients, PE was associated with deep vein thrombosis. $16.6 \%$ of patients had a shock index $\geq 1$ and $84.75 \%$ simplified pulmonary embolism severity index (PESI) score $\geq 1$. A number of $51.5 \%$ of patients presented echocardiographic right heart dysfunction, $50.6 \%$ and $55.9 \%$ of patients presented increased values of troponins and natriuretic peptides, respectively. The following percentage, $6.5 \%, 71.1 \%$ and $22.4 \%$, were defined as high, intermediate and low risk according to the European Society of Cardiology (ESC) criteria, respectively. Antithrombotic treatment was started in Internal Medicine wards in $60.6 \%$ of patients. $4.9 \%$ of patients were treated with systemic thrombolysis, $15.1 \%$ with intravenous unfractionated heparin, whereas low molecular weight heparins and fondaparinux were performed in $39.2 \%$ and $39.8 \%$ of patients, respectively. Vitamin K antagonists were prescribed in $52.1 \%$ of patients at hospital discharge. Fatal and non-fatal major bleedings occurred in $1.7 \%$ of patients. TUSCAN-PE study contributes to the knowledge of real life management of acute PE in the Internal Medicine setting.
\end{abstract}

Correspondence: Luca Masotti, UO Medicina Interna, Ospedale Santa Maria Nuova, Piazza Santa Maria Nuova 1, 50123 Firenze, Italy. E-mail: luca.masotti@tin.it

Key words: Pulmonary embolism; deep vein thrombosis; biomarkers; diagnosis; prognosis; therapy.

Conflict of interest: the authors declare no potential conflict of interest.

See online Appendix for Members of the TUscan Study on Characteristics ANd clinical management of Pulmonary Embolism in internal medicine wards (TUSCAN-PE) Investigators.

Received for publication: 20 March 2014.

Accepted for publication: 24 March 2014.

This work is licensed under a Creative Commons Attribution NonCommercial 3.0 License (CC BY-NC 3.0).

CCopyright L. Masotti et al., 2015

Licensee PAGEPress, Italy

Italian Journal of Medicine 2015; 9:125-133

doi:10.4081/itjm.2014.504

\section{Introduction}

Acute pulmonary embolism (PE) remains one of the leading causes of mortality and morbidity in cardiovascular setting. In the United States, about 150,000 patients per year are diagnosed with acute PE, resulting in thousands of deaths annually. ${ }^{1}$ In Italy epidemiological studies estimated a possible incidence of 30-100/100,000 inhabitants/year. ${ }^{2-4}$ In Tuscany, one of the most populated regions of Italy encompassing for more than 3.7 million of inhabitants, around 2650 cases of PE were diagnosed in the first six diagnoses of the hospital discharge schedules in 2009 and 1861 diagnosis related groups with number 078 were registered, burdening for $0.40 \%$ of overall hospital admissions. ${ }^{5}$ Mortality for PE is strictly dependent on hemodynamic compromise at presentation, ranging from less than $3 \%$ in normotensive patients without evidence of right ventricular dysfunction (RVD) to up to $30 \%$ in patients with shock and up to $70 \%$ in pa- 
tients with cardiac arrest at presentation. ${ }^{1}$ In Tuscany, in 2009 the in-hospital mortality in patients with diagnosis of acute PE was $13.2 \%$, encompassing for $2.1 \%$ of overall in-hospital mortality. ${ }^{5}$

Many clinical studies or registries are available for defining clinical characteristics and management of acute PE. However many of these have been performed in the settings of Cardiology, Emergency Medicine or Respiratory Diseases settings, such as the PIOPED I and II, MAPPET, ICOPER, the more recently published IPER and EMPEROR or the oldest PISA-PED. ${ }^{6-12}$ Furthermore other studies or registries such as MASTER, RIETE and the ongoing SCOPE, despite enclosing Italian Internal Medicine Units, were conducted in Centers of recognized experience in the field of venous thromboembolism, whereas other studies such as ZATPOL and SWIVTER were conducted in European countries out of Italy. ${ }^{13-17}$

Practical recommendations on prevention, diagnosis, prognostic stratification and acute treatment have had wide diffusion in the last years ${ }^{18-21}$ but evidence about their application in real life lack. Therefore the aim of our study was to provide information about characteristics and clinical management of acute PE patients admitted in the setting of Italian Internal Medicine wards.

\section{Materials and Methods}

$T U$ scan Study on Characteristics ANd clinical management of Pulmonary Embolism in internal medicine wards (TUSCAN-PE) study was a multicenter, observational, retrospective, cohort study aimed to evaluate characteristics and clinical management of acute PE patients admitted in Internal Medicine wards of Tuscany affiliated to the Italian Federation of Associations of Hospital Doctors on Internal Medicine (FADOI).

Each center was invited to create a database referred to at least ten patients consecutively discharged for acute PE in 2012. Database was made aimed to provide information about demographic, clinical aspects, outcome, risk factors for venous thromboembolism (VTE), pharmacological prophylaxis before admission, diagnostic tools, prognostic stratification, therapeutic management and its bleeding complications during hospital stay. Data were collected in the first three months of 2013 and accordingly submitted to head Center, that provide for final analysis.

\section{Results}

A total of 452 patients from 28 Internal Medicine Units representatively allocated over the whole Tuscany (Figure 1) $(60.2 \% \mathrm{~F})$, with mean age $76.01 \pm 12.34$ years, were enrolled. General characteristics of patients are summarized in Table 1. Fifteen Centers were affiliated to primary non-academic Hospitals, 9 Centers to secondary non-academic Hospitals and 4 Centers to tertiary academic Hospitals.

A percentage of $87 \%$ of patients was admitted from Emergency Department, 8\% from Cardiology or Intensive wards, $2 \%$ from surgical wards and $3 \%$ from other hospital wards. $67.9 \%$ of patients $(65.2 \%$ of those admitted from Emergency Department) was admitted with main diagnosis of PE, whereas $32.1 \%$ of patients (34.8\% of those admitted from Emergency Department) was admitted with another diagnosis and the diagnosis of PE was performed by Internists. The main alternative diagnoses are showed in Table 2. The mean time from hospital arrival to confirmed diagnosis of $\mathrm{PE}$ was $1.34 \pm 3.70$ days (range $0-51$ days). In patients in whom Internists made diagnosis of PE, the mean time from hospital admission to confirmed diagnosis was 2 days (mean 3.15 \pm 3.90 days, range 0-30 days). In patients in whom diagnosis of PE was searched and made by Internists, the mean time from hospital admission to diagnosis was 1 day. In $78.5 \%$ of patients, diagnosis was performed by computer tomography pulmonary angiography (CTPA) scan alone, in $5.1 \%$ by using scintigraphy lung scan alone, in $7.5 \%$ by combination of CTPA and scintigraphy lung scan. In $8.9 \%$ of patients diagnosis was performed based on clinical suspicion associated with detection of deep vein thrombosis at legs ultrasonography and/or echocardiographic or biomarkers signs of right heart dysfunction. A percentage

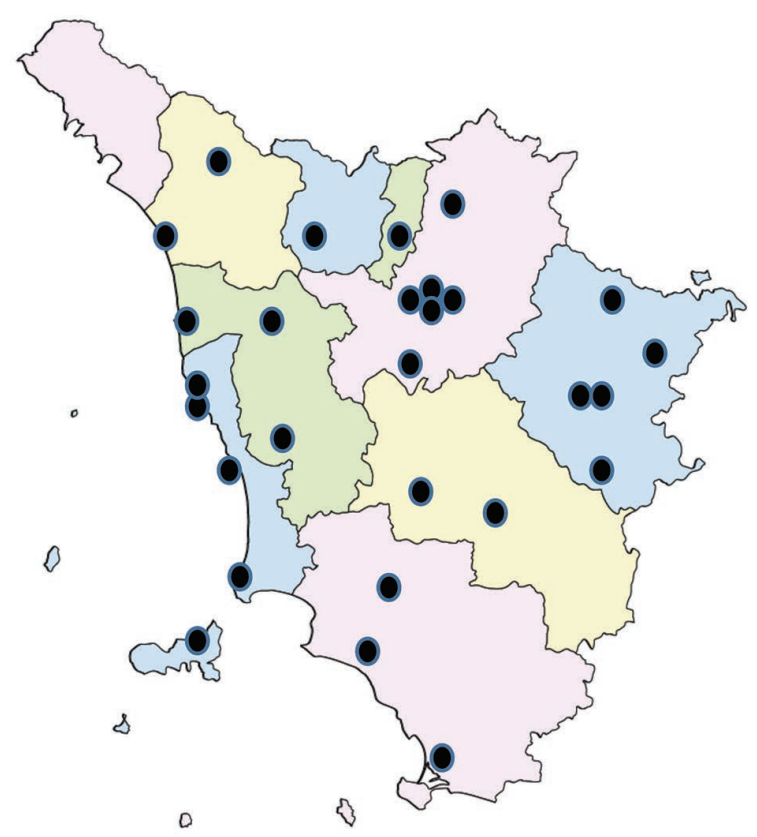

Figure 1. TUSCAN-PE Centers. 
of $14.8 \%$ of PE diagnoses was incidental, $22.6 \%$ when performed by internists. In patients in whom diagnosis of PE was performed by CTPA, $49.6 \%$ of thromboemboli were central (main pulmonary or its branches), $20.8 \%$ lobar, $29.6 \%$ segmental or sub-segmental. In $61.5 \%$ of cases PE was bilateral. In patients in whom PE was found by scintigraphy lung scan, $76 \%$ of cases presented 2 or more perfusion defects. Legs ultrasonography was performed in $83.6 \%$ of patients. In $65.8 \%$ of patients, a deep vein thrombosis (DVT) was found, $45.2 \%$ proximal and $20.6 \%$ distal. DVT was bilateral in $16.8 \%$ of patients.

Recent (within 3 months) respiratory tract infections (55.3\%), immobility (42.25\%), hospital admissions within the last three months (overall $33.6 \%$, medical setting $25.8 \%$, surgical setting $7.8 \%$ ) and cancer $(30.3 \%)$ were the main risk factors for VTE. Figure 2 summarizes the risk factors for VTE. 33\% of patients performed VTE prophylaxis before hospital admission, $13.1 \%$ full prophylactic dose of low molecular weight heparins (LMWHs) or fondaparinux, $15.6 \%$ reduced prophylactic dose of LMWHs or fondaparinux, $4.2 \%$ vitamin $\mathrm{K}$ antagonists $(2.2 \%$ under dosed). All-cause mortality was $9.5 \%$, whereas $5.75 \%$ was PE-related. A percentage of $16.6 \%$ of patients had a severity index (SI) $\geq 1$. All-cause mortality was $6.10 \%$ (3.70\% PE related) in patients with $\mathrm{SI}<1$ versus $25.30 \%$ (17.30\% PE related) in patients with SI $\geq 1.84 .75 \%$ of patients had a simplified pulmonary embolism shock index (sPESI) score $\geq 1$. All-cause and PE-related mortality were $0 \%$ in patients with sPESI score 0, whereas $10.95 \%$ and $5.75 \%$ were respectively in patients with sPESI score $\geq 1$.

Trans-thoracic echocardiogram, natriuretic peptides [brain natriuretic peptide (BNP) or its terminal portion (NT-proBNP)] and troponins assays were performed respectively in $70.1 \%, 35.6 \%$ and $80.7 \% .51 .5 \%$ of patients undergone to trans-thoracic echocardiography showed RVD, whereas $50.6 \%$ and $55.9 \%$ of patients respectively undergone to BNP/NT-proBNP and troponins assays presented increased values of these. The European Society of Cardiology (ESC) criteria defining patients in high, intermediate and low early mortality risk were applicable to $78.4 \%$ of patients. ${ }^{18}$ Figure 3 shows the early mortality risk distribution according to ESC criteria and in-hospital mortality according to these in the analyzed patients. Antithrombotic treatment was started in Internal Medicine wards in $60.6 \%$ of patients. A percentage of $4.9 \%$ of patients underwent intravenous thrombolysis, $15.1 \%$ to intravenous unfractionated heparin, whereas LMWHs and fondaparinux were performed in $39.2 \%$ and $39.8 \%$, respectively. In $1.1 \%$ of patients, a vena cava filter was placed. Table 3 shows the acute treatment according to early mortality risk assessed by ESC criteria. Treatment related bleedings occurred in $3.1 \%$ of patients, $0.2 \%$ being fatal, $1.5 \%$ major and $1.4 \%$ non-major. Table 4 summarizes bleeding complications.

At hospital discharge, vitamin $\mathrm{K}$ antagonists were prescribed in $52.1 \%$ of patients, whereas LMWHs and fondaparinux were prescribed in $24.9 \%$ and $21.8 \%$ of patients, respectively.

Table 1. General characteristics.

\begin{tabular}{lc}
\hline Patients number & 452 \\
\hline Males/Females & $180 / 272$ \\
\hline Mean age (years) & $76.01 \pm 12.34$ \\
Males & $73.55 \pm 12.45$ \\
Females & $77.65 \pm 12.02$ \\
Over 75 years & $62.70 \%$ \\
Over 85 years & $28.1 \%$ \\
\hline Mean hospital stay (days) & $10.60 \pm 6.63$ \\
\hline In-hospital mortality & \\
Overall & $9.50 \%$ \\
PE related & $5.75 \%$ \\
\hline VTE prevention before admission & $33 \%$ \\
Full prophylactic dose LMWHs or fondaparinux & $13.1 \%$ \\
Reduced prophylactic dose LMWHs or fondaparinux & $15.6 \%$ \\
VKAs & $4.2 \%$ \\
Under dosed & $2.2 \%$ \\
In range & $1.5 \%$ \\
Over dosed & $0.5 \%$ \\
\hline Mean heart rate (beats for minute) & $92.37 \pm 20.26$ \\
\hline Mean systolic blood pressure (mmHg) & $127.45 \pm 24.36$ \\
\hline Mean PaO $(\mathrm{mmHg})$ & $63.7 \pm 13.8$ \\
\hline Renal failure & $20.6 \%$ \\
Moderate (CrCl 30-50 mL/min) & $6 \%$ \\
\hline Severe ( $<30$ mL/min) & \\
\hline
\end{tabular}

PE, pulmonary embolism; VTE, venous thromboembolism; LMWHs, low molecular weight heparins; VKAs, vitamin K antagonists.

Table 2. Main alternative diagnoses at hospital admission.

\begin{tabular}{lc}
\hline Pulmonary embolism mimics & $64.3 \%$ \\
\hline Pulmonary infiltrate & $14.7 \%$ \\
\hline Pleural effusion & $14.7 \%$ \\
\hline Respiratory failure & $10.4 \%$ \\
\hline Heart failure & $7.8 \%$ \\
\hline Syncope & $6.1 \%$ \\
\hline Chest pain & $2.6 \%$ \\
\hline Chest pain associated with syncope & $1.7 \%$ \\
\hline Fever & $1.7 \%$ \\
\hline Deep vein thrombosis as main diagnosis & $10.4 \%$ \\
\hline Other diagnoses & $25.3 \%$ \\
\hline
\end{tabular}




\section{Discussion}

Acute PE in one of the most feared diseases to manage in Internal Medicine wards, due to high mortality and morbidity. Prevention, diagnosis, prognostic stratification and treatment represent the mainstays of its hospital management and remain challenges for physicians. The burden of VTE, of which PE represents the most feared manifestation, is so severe in acutely ill medical patient that to date around $75 \%$ of the diagnoses of VTE in hospitalized patients is referred to medical patients and $75 \%$ of VTE-related deaths occurs in this kind of subjects. ${ }^{22-26}$ Despite recommendations on PE management are worldwide widespread, literature data on how physicians translate these in real practice lack. Therefore studies aimed to give information about real life management of acute PE are warranted. TUSCAN-PE study aimed to give answers about it in the context of Internal Medicine wards of Tuscany.

Findings from our study demonstrates that the PE population discharged from Internal Medicine wards is very old, more than $60 \%$ being 75 years-old and older and around $30 \% 85$ years-old and older. Compared with other studies, the population of TUSCAN-PE is older, even when considered other Italian populations enrolled in registries, such as those of IPER, an Italian registry performed in 49 Centers of which 37 Cardiology Units, 6 Emergency Medicine Units and 6 Internal Medicine Units, and RIETE, an international multicenter registry. ${ }^{6-14,27}$ One of the most important findings emerged in TUSCAN-PE study was that around one third of patients received diagnosis of PE from Internists in the first hours from admission in their wards. Diagnosis of acute PE remains a challenge due to non-specific presentation in terms of signs, instrumental and laboratory findings, especially in the older patients such as those of our study. ${ }^{28}$ Physicians should increase their efforts to reduce misdiagnoses and diagnostic delays. Clinical suspicion should be the primum movens for diagnosis of acute PE and the estimation of pre-test probability (PTP) by using validated tools, such as Wells or Geneva scores, may rise it. Therefore their use in clinical practice should be encouraged..$^{29,30} \mathrm{CTPA}$ is now recognized as the main and most available diagnostic tool to rule in the diagnosis of acute PE. ${ }^{18-20}$ Strategies based on likely PTP and/or positive D-dimer identify patients undergoing CTPA or scintigraphy lung scan aimed to rule in the diagnosis of PE. ${ }^{18-20}$ Legs ultrasonography permits to detect DVT which represents the major font of PE. ${ }^{18-20}$ Legs ultrasonography could be enclosed in the diagnostic work-up of PE, since than the presence of proximal DVT in patients with likely PTP scoring may

Table 3. Treatment according to European Society of Cardiology criteria-based mortality risk.

\begin{tabular}{lccc}
\hline & High risk (\%) & Intermediate risk (\%) & Low risk (\%) \\
\hline rtPA & 13.6 & 6.4 & 1.2 \\
\hline UFH & 27.7 & 17.2 & 15.1 \\
\hline LMWH or fondaparinux & 58.7 & 76,4 & 83.7 \\
\hline
\end{tabular}

UFH, unfractionated heparin; LMWH, low molecular weight heparins.

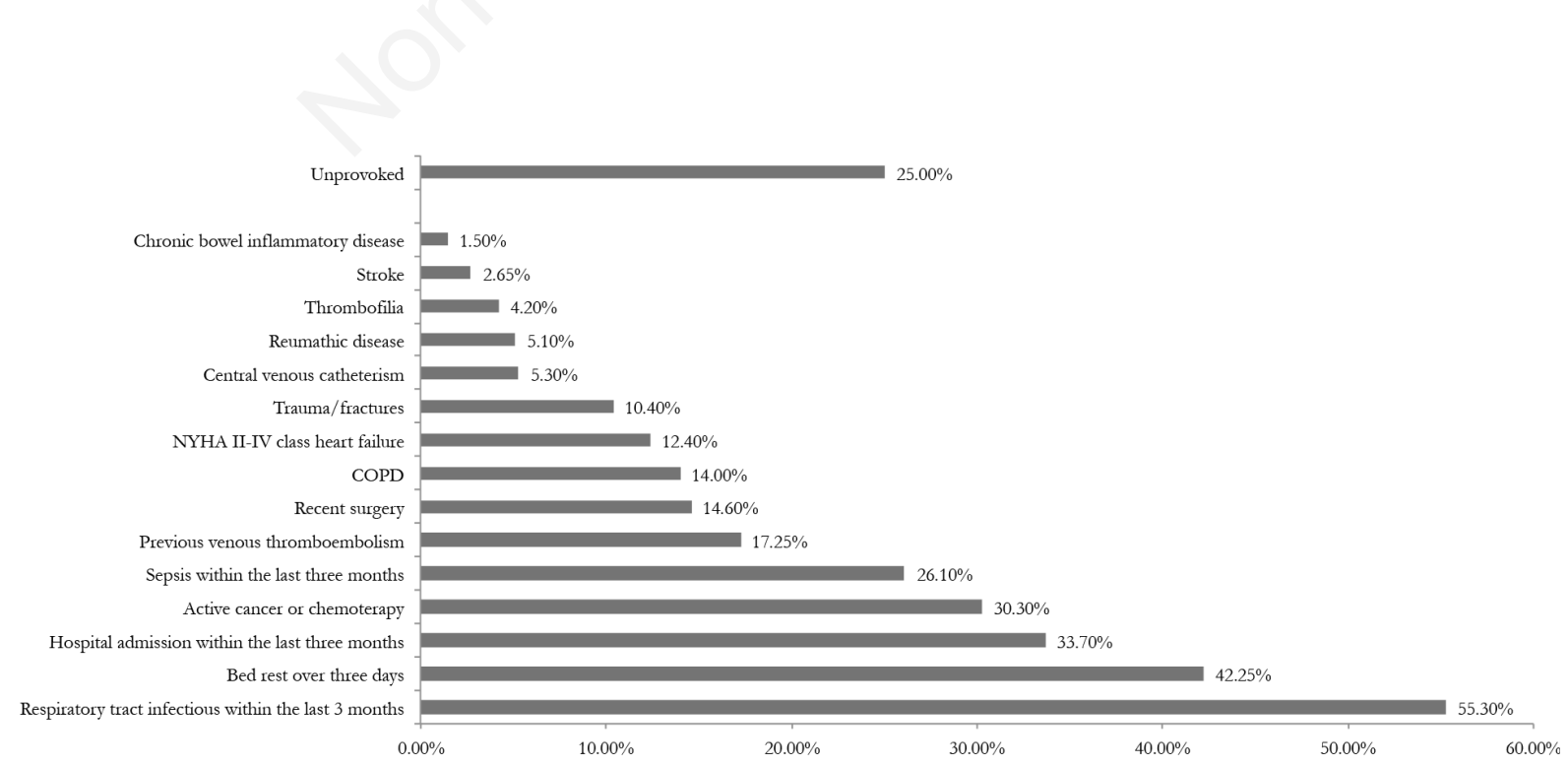

Figure 2. Risk factors for venous thromboembolism. COPD, chronic obstructive pulmonary disease. 
rule in indirectly the diagnosis and conclude the diagnostic process. ${ }^{18-20}$ Our study demonstrates that to date CTPA is the most widespread diagnostic tool for PE, whereas only few patients undergo scintigraphy lung scan. These findings agree with those of most recent studies. ${ }^{10,11}$ Moreover our study demonstrates that legs ultrasonography for DVT detecting is routine practice in Internal Medicine.

The main risk factors for VTE in ill medical patients are represented by stroke, heart failure, cancer, chronic obstructive pulmonary disease associated with respiratory failure (especially when requiring mechanical ventilation), acute myocardial infarction, sepsis, rheumatic and chronic inflammatory bowel diseases. The risk increases significantly when these conditions occur in elderly subjects aged $>75$ years, in patients with impaired mobility, those with a history of previous VTE and those with inherited or acquired thrombophilia. ${ }^{22,31-34}$ In TUSCAN-PE recent infectious diseases, especially affecting the respiratory system, were found as the main risk factors of PE. Despite this percentage is much greater compared to the most recently published studies on topic, ${ }^{10,11,14,17}$ this finding is not completely surprising, since than in the recent years many literature reports have focused on link between infectious diseases, inflammation and thrombosis. ${ }^{35,36}$ Further investigations are warranted to better define the role of infectious diseases as risk factors for PE. Immobility remains one of the most frequent risk factors for VTE, especially in elderly patients and our study confirms it in agreement with literature. ${ }^{9-14,17,28}$

In TUSCAN-PE around one third of patients discharged with PE from Internal Medicine wards has active cancer. This finding is higher compared to the most

Table 4. Treatment related bleedings.

\begin{tabular}{ll}
\hline Overall & $3.1 \%$ \\
\hline Fatal bleedings (brain) & $0.2 \%$ \\
\hline Non-fatal major bleedings & $1.5 \%$ \\
\hline Brain & $0.2 \%$ \\
\hline Retroperitoneal & $0.2 \%$ \\
\hline Gastro-intestinal & $1.1 \%$ \\
\hline Non-major bleedings & $1.4 \%$
\end{tabular}

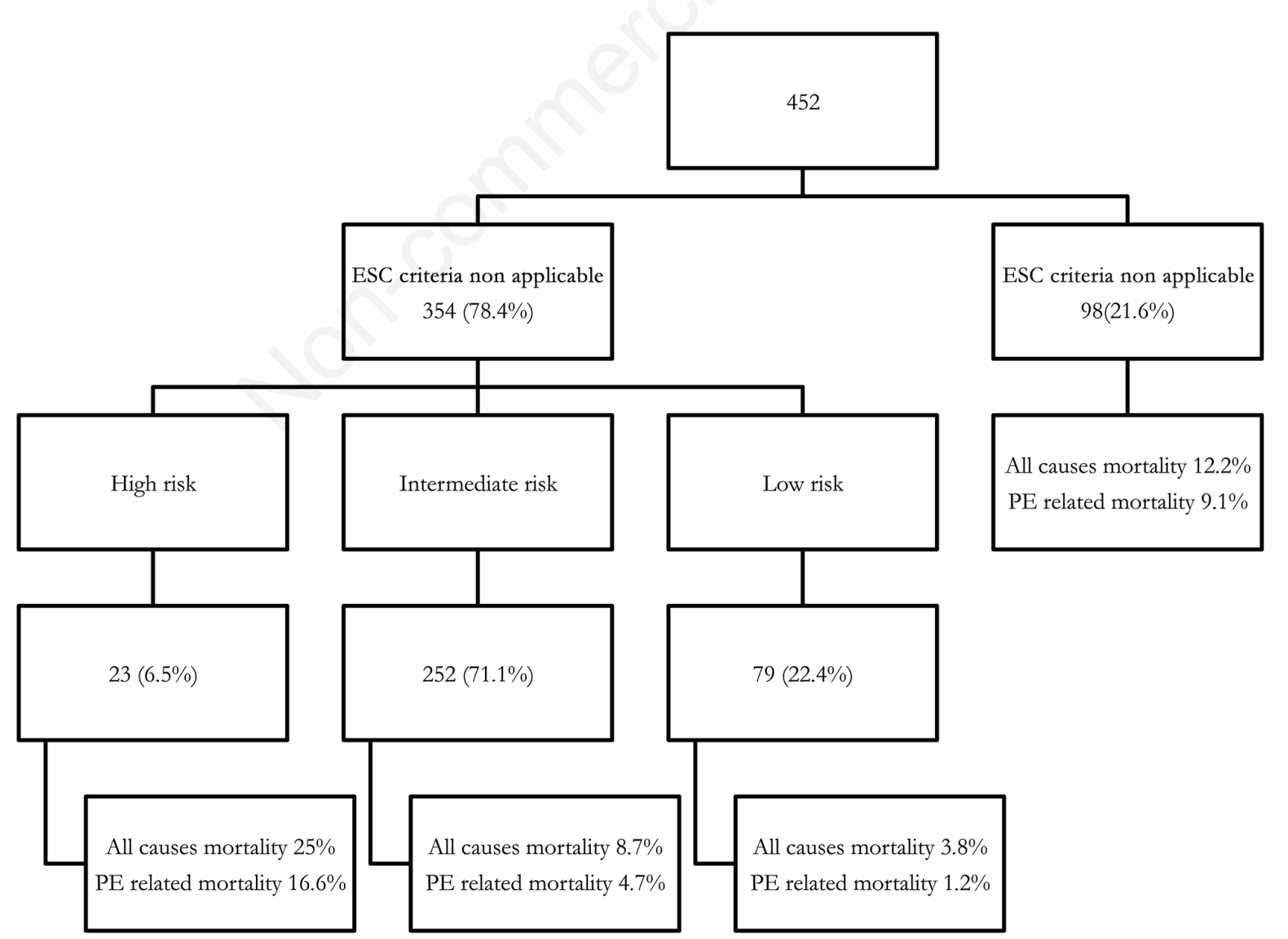

Figure 3. Risk stratification according to European Society of Cardiology (ESC) criteria. PE, pulmonary embolism. 
recent studies, such as IPER, EMPEROR, MASTER, SWIVTER and ZATPOL, but similar to that observed in the Italian population of RIETE registry. ${ }^{10,11,13,16,17,27}$ This could be ascribed to higher mean age of patients enrolled in our study other than a possible major selection of patients admitted in non-Internal Medicine settings, such as those of the abovementioned studies.

Recent hospitalization as possible risk factor for VTE was already underlined from Spencer et al. in 2007 in the United States and more recently has been found in the EMPEROR and SWIVTER studies. ${ }^{11,17,37}$ In the study of Spencer et al., the burden of recent hospitalization in surgical or medical settings was similar, whereas in our study $76.7 \%$ of patients with $\mathrm{PE}$ who had been recently admitted to hospital were in medical setting. In the EMPEROR study $23.8 \%$ of patients with PE had been recently hospitalized, but it's unclear the setting of hospitalization. ${ }^{11}$

Randomized controlled trials have clearly demonstrated the efficacy and safety of pharmacological prophylaxis in ill medical patient. ${ }^{22,38-40}$ A meta-analysis of literature evidence demonstrated that VTE pharmacological prophylaxis during hospital stay in ill-medical patients significantly reduces the incidence of DVT, non-fatal and fatal PE during hospital stay without increasing the risk of major bleeding, but any advantage on overall mortality was found. ${ }^{41}$ Studies aimed to analyze the role of extended pharmacological prophylaxis in ill medical patients have found a low risk/benefit ratio due to not acceptable rate of bleedings compared to placebo. ${ }^{42-44}$ Therefore the most recent guidelines suggest to avoid prolonged pharmacological thromboprophylaxis in ill medical patients beyond two weeks. ${ }^{45}$ However, as seen, the recent hospitalization as possible risk factors for $\mathrm{PE}$ is a cumbersome problem. Avoiding to prolong prevention could be a risk in subgroups of patients, such as patients suffering from respiratory tract infections or sepsis or confined to bed. In the SWIVTER study 58\% of patients developing VTE episodes during or shortly after hospitalization had received prophylaxis, these findings being similar to those observed in our study. ${ }^{46}$ Therefore extended prophylaxis and its dose should be tailored on single patient. In TUSCAN-PE in fact many patients with acute $\mathrm{PE}$ were on pharmacological prophylaxis at hospital arrival, even if at lower doses in one half of patients undergone to pharmacological prophylaxis despite moderate or severe renal failure were found only in $26 \%$ of this subgroup. In 2008 ESC suggested to divide PE patients in three classes at different early mortality risk based on hemodynamic compromise at presentation. ${ }^{18}$ Patients with acute PE presenting with cardiac arrest, shock [systolic blood pressure (SBP) $\leq 90 \mathrm{mmhgHg}$ ] or a drop of 40 $\mathrm{mmHg}$ in SBP respect the usual for at least $15 \mathrm{~min}$ were defined as high risk being early mortality in these patients higher than $15 \%$, whereas normotensive patients with signs of RVD or increased troponins as sign of myocardial damage were defined at intermediate risk, early mortality ranging from $15 \%$ to $3 \%$, and finally normotensive patients without RVD or myocardial damage were defined at low risk, early mortality being lower than $1 \% .{ }^{18} \mathrm{ESC}$ suggested that acute treatment should be tailored on the basis of early mortality risk. Therefore in acute phase of treatment, high risk patients should receive intravenous thrombolysis if not contraindicated or embolectomy, whereas intermediate risk patients and low risk patients should receive unfractionated heparin (UFH), LMWHs or fondaparinux. LMWHs or fondaparinux should be preferred over UFH with the exception of patients with severe renal failure. Oral anticoagulation with vitamin $\mathrm{K}$ antagonists (VKA) should be overlapped to parenteral anticoagulants starting from the first day. ${ }^{18}$ In low risk patients, ESC recommendations suggested early hospital discharge or at home treatment. ${ }^{18}$ ESC criteria were derived from available literature in that moment and on Expert consensus, but a validation from clinical trials was lacking. ${ }^{18}$

Prognostic stratification of early risk mortality is therefore of utmost importance. ${ }^{47}$ Other than ESC criteria, prognostic stratification could be performed by using clinical parameters or clinical scoring, such as the SI or the SPESI. ${ }^{47,48}$ SI consider the ratio between heart rate (HR) in beat for minutes (bpm) and SBP in $\mathrm{mmHg}$. High risk of early mortality is defined by $\mathrm{SI} \geq 1 .{ }^{47}$ The sPESI score considers variables associated with early mortality risk and derived from multivariate analysis, such as age over 80 years, history of cancer, heart failure and/or chronic obstructive pulmonary diseases, $\mathrm{HR}>110 \mathrm{bpm}, \mathrm{SBP}<100 \mathrm{mmHg}$, arterial oxygen saturation $\left(\mathrm{SaO}_{2}\right)<90 \%$ or arterial oxygen partial pressure $\left(\mathrm{PaO}_{2}\right)<60 \mathrm{mmHg}$. sPESI score ranges from 0 to 6 . In the validation cohort, early mortality rate was lower than $1 \%$ in patients with sPESI score 0 and higher than $10 \%$ in patients with sPESI score $\geq 1 .{ }^{48}$

Despite data were collected retrospectively, our study highlights the importance of prognostic stratification of acute PE in clinical practice. ESC criteria, SI and SPESI permit to stratify prognosis of PE in real life. Our study in fact validated the ESC criteria in real practice, as more recently occurred with the IPER study. ${ }^{49}$ In-hospital mortality rates in fact fell into those speculated from ESC recommendations.

Our study found that SI really identified high mortality risk patients, but unfortunately it didnot identify low risk patients. Therefore it may be used to select patients needing for closer monitoring and more aggressive therapy but it should not be used alone for identifying patients candidate to early discharge or at home treatment.

Finally our study contributes to the external vali- 
dation of the SPESI as prognosticator. Our study confirms that sPESI is highly sensitive to identify low risk patients. Patients with sPESI score 0 in fact had an inhospital mortality rate of $0 \%$. Therefore sPESI may be an optimal instrument to identify patients candidate for early hospital discharge or at home treatment.

Our study highlights the low percentage $(4.9 \%)$ of patients undergone to systemic thrombolysis despite high early mortality risk. In the IPER study, $11 \%$ of patients underwent to systemic thrombolysis, but it reached $40 \%$ in unstable patients versus $13.6 \%$ in this kind of patients in our study. ${ }^{10}$ In the SWIVTER study 3.3\% of patients underwent to systemic thrombolysis, whereas in the EMPEROR study systemic thrombolysis was performed in $2.3 \%$ of patients. ${ }^{11,17}$ The reason for the underuse of thrombolysis may be ascribed to the fear of bleeding complications in an old and fragile population such as that admitted in Internal Medicine wards, even if advanced age alone should not be considered as a contraindication for this treatment option. High prevalence of immobilized and cancer patients encountered in our study may define a fragile population.

Our study demonstrates that invasive procedures such as pulmonary embolectomy or vena cava filter placement, despite recommended as emergency procedure in patients with absolute contraindication to anticoagulation or in the case of embolectomy not responding to it, are rarely performed, probably due to the lack of availability, especially in most peripheral or non-academic Centers. ${ }^{18,19,50}$

On the other side our study highlights the high percentage of patients treated with LMWHs or fondaparinux in acute phase, even if in high risk patients, performed in more than $80 \%$ of overall patients, in agreement with literature evidence. ${ }^{10,11,14,16}$ Surprisingly, around one half of our patients were discharged without switching to oral anticoagulation. However, it is not possible to know if this kind of therapy has been started after hospital discharge due to the lack of follow-up. The main limitations of vitamin $\mathrm{K}$ antagonists, especially in elderly patients, such as unpredictable pharmacological profile and narrow therapeutic window requiring routine laboratory monitoring, slow onset and offset of action, multiple drug and food interactions and the fear of bleeding complications may make physicians reluctant to use VKA. These findings agree with those of RIETE registry agree, demonstrating that parenteral anticoagulation seems to be the choice treatment option in very old patients, such as nonagenarians..$^{51}$

The marketing of new oral anticoagulants, safer and easier to use in the acute phase of PE, may increase the percentage of patients switched to oral anticoagulation, but to date literature evidence on real life management of PE in the era of new oral anticoagulants lacks. $^{52}$
Treatment related bleedings represent an independent risk factor of mortality in acute phase of PE. ${ }^{53}$ The prevalence of bleedings in our study agrees with literature evidence. ${ }^{10-14,16,17}$

\section{Conclusions}

The approach to diseases in real world is of utmost importance for planning care. TUSCAN-PE focuses on real life management of acute PE in Internal Medicine wards of Tuscany, independently from hospital level and grading of expertise on this topic. Findings from TUSCAN-PE study demonstrate that: i) diagnosis of $P E$ remains a challenge; ii) some risk factors such as respiratory infectious diseases and sepsis are emerging; iii) recent hospitalization in medical setting may represent a trigger for the development of procoagulative mechanisms which lead to PE despite pharmacological prophylaxis; iv) prognostic stratification allows to define the early mortality risk; but v) treatment is far to be tailored on it due to frailty of patients in the setting of Internal Medicine wards. Prospective studies are warranted to better define these findings.

\section{References}

1. Wood KE. Major pulmonary embolism. Review of a pathophysiologic approach to the golden hour of hemodinamically significant pulmonary embolism. Chest 2002;121:877-905.

2. Giuntini C, Di Ricco G, Marini C, et al. Pulmonary embolism: epidemiology. Chest 1995;107:3S-9S.

3. Roncon L, Zonzin P, Giommi L, et al. The diagnostic and therapeutic procedures in pulmonary embolism: a survey in the Veneto Region. Cardiologia 1999;44:735-41.

4. Zonzin P, Agnelli G, Casazza F, et al. Comments on the guidelines of the European Society of Cardiology Task Force on pulmonary embolism. Ital Heart J Suppl 2001;2:1342-56.

5. Masotti L, Pampana A, Pocobelli B, Landini GC. Incidence, hospital burden and diagnostic approach of acute pulmonary embolism in a Tuscan province. Blood Transfus 2010;8:s59.

6. PIOPED Investigators. Value of the ventilation/perfusion scan in acute pulmonary embolism. Results of the prospective investigation of pulmonary embolism diagnosis (PIOPED). JAMA 1990;263:2753-9.

7. Stein PD, Fowler SE, Goodman LR, et al. Multidetector computed tomography for acute pulmonary embolism. N Engl J Med 2006;354:2317-27.

8. Kasper W, Konstantinides S, Geibel A, et al. Management strategies and determinants of outcome in acute major pulmonary embolism: results of a multicenter registry. J Am Coll Cardiol 1997;30:1165-71.

9. Goldhaber SZ, Visani L, De Rosa M. Acute pulmonary embolism: clinical outcomes in the International Cooperative Pulmonary Embolism Registry (ICOPER). Lancet 1999;353:1386-9. 
10. Casazza F, Becattini C, Bongarzoni A, et al. Clinical features and short term outcomes of patients with acute pulmonary embolism. The Italian Pulmonary Embolism Registry (IPER). Thromb Res 2012;130:847-52.

11. Pollack CV, Schreiber D, Goldhaber SZ, et al. Clinical characteristics, management, and outcomes of patients diagnosed with acute pulmonary embolism in the emergency department: initial report of EMPEROR (Multicenter Emergency Medicine Pulmonary Embolism in the Real World Registry). J Am Coll Cardiol 2011; 57:700-6.

12. Miniati M, Pistolesi M, Marini C, et al. Value of perfusion lung scan in the diagnosis of pulmonary embolism: results of the Prospective Investigative Study of Acute Pulmonary Embolism Diagnosis (PISA-PED). Am J Respir Crit Care Med 1996;154:1387-93.

13. Agnelli G, Verso M, Ageno W, et al. The MASTER registry on venous thromboembolism: description of the study cohort. Thromb Res 2008;121:605-10.

14. Monreal M, Suárez C, Fajardo JA, et al. Management of patients with acute venous thromboembolism: findings from the RIETE registry. Pathophysiol Haemost Thromb 2003-2004;33:330-4.

15. Study on the clinical course of pulmonary embolism (SCOPE). Available from: http://www.clinicaltrials. gov/ct2/show/NCT01781858?term=scope+AND+embolism\&rank=1

16. Budaj-Fidecka A, Kurzyna M, Fijałkowska A, et al. Inhospital major bleeding predicts mortality in patients with pulmonary embolism: an analysis of ZATPOL Registry data. Int J Cardiol 2013;168:3543-9.

17. Spirk D, Husmann M, Hayoz D, et al. Predictors of inhospital mortality in elderly patients with acute venous thrombo-embolism: the SWIss Venous ThromboEmbolism Registry (SWIVTER). Eur Heart J 2012;33:921-6.

18. Torbicki A, Perrier A, Konstantinides S, et al. Guidelines on the diagnosis and management of acute pulmonary embolism of the European Society of Cardiology. Eur Heart J 2008;29:2276-315.

19. National Clinical Guideline Centre (UK). Venous thromboembolic diseases: the management of venous thromboembolic diseases and the role of thrombophilia testing [Internet]. London: Royal College of Physicians; 2012.

20. Jaff MR, McMurtry MS, Archer SL, et al. Management of massive and submassive pulmonary embolism, iliofemoral deep vein thrombosis, and chronic thromboembolic pulmonary hypertension: a scientific statement from the American Heart Association. Circulation 2011;123:1788-830.

21. Kearon C, Akl EA, Comerota AJ, et al. Antithrombotic therapy for VTE disease: antithrombotic therapy and prevention of thrombosis, 9th ed: American College of Chest Physicians Evidence-Based Clinical Practice Guidelines. Chest 2012;141:e419S-94S.

22. Samama MM, Kleber FX. An update on prevention of venous thromboembolism in hospitalized acutely ill medical patients. Thromb J 2006;4:8.

23. Moheimani F, Jackson DE. Venous thromboembolism: classification, risk factors, diagnosis, and management. ISRN Hematol 2011;ID 124610.

24. Geerts WH, Bergqvist D, Pineo GF, et al. Prevention of venous thromboembolism: American College of Chest Physicians evidence-based clinical practice guidelines (8th Edition). Chest 2008;133:381-453.
25. Alikhan R, Cohen AT, Combe S, et al. Risk factors for venous thromboembolism in hospitalized patients with acute medical illness: analysis of the MEDENOX Study. Arch Intern Med 2004;164:963-8.

26. Goldhaber SZ, Dunn K, MacDougall RC. New onset of venous thromboembolism among hospitalized patients at Brigham and Women's Hospital is caused more often by prophylaxis failure than by withholding treatment. Chest 2000;8:1680-4.

27. Di Micco P, Bura-Riviere A, Poggio R, et al. Clinical characteristics of Italian patients with venous thromboembolism enrolled in the RIETE Registry. Ital J Med 2011;5:255-60.

28. Masotti L, Ray P, Righini M, et al. Pulmonary embolism in the elderly: a review on clinical, instrumental and laboratory presentation. Vasc Health Risk Manag 2008;4: 629-36.

29. Writing Group for Christopher Study Investigators. Effectiveness of managing suspected pulmonary embolism using an algorithm combining clinical probability, Ddimer testing, and computer tomography. JAMA 2006; 295:172-9.

30. Le Gal G, Righini M, Roy PM, et al. Prediction of pulmonary embolism in the emergency department: the revised Geneva score. Ann Intern Med 2006;144:165-71.

31. Geerts WH, Bergqvist D, Pineo GF, et al. Prevention of venous thromboembolism: American College of Chest Physicians evidence-based clinical practice guidelines (8th Edition). Chest 2008;133:381-453.

32. Alikhan R, Cohen AT, Combe S, et al. Risk factors for venous thromboembolism in hospitalized patients with acute medical illness: analysis of the MEDENOX Study. Arch Intern Med 2004;164:963-8.

33. Goldhaber SZ, Dunn K, MacDougall RC. New onset of venous thromboembolism among hospitalized patients at Brigham and Women's Hospital is caused more often by prophylaxis failure than by withholding treatment. Chest 2000;8:1680-4.

34. Alikhan R, Peters F, Wilmott R, et al. Fatal pulmonary embolism in hospitalized patients: a necropsy review. J Clin Pathol 2004;57:1254-7.

35. Levine RL, LeClerc JR, Bailey JE, et al. Venous and arterial thromboembolism in severe sepsis. Thromb Haemost 2008;99:892-8.

36. Clayton TC, Gaskin M, Meade TW. Recent respiratory infection and risk of venous thromboembolism: case control study through a general practice database. Int $\mathrm{J}$ Epidemiol 2011;40:819-27.

37. Spencer FA, Lessard D, Emery C, et al. Venous thromboembolism in the outpatient setting. Arch Intern Med 2007; 167:1471-5.

38. Samama MM, Cohen AT, Darmon JY, et al. A comparison of enoxaparin with placebo for the prevention of venous thromboembolism in acutely ill medical patients. Prophylaxis in Medical Patients with Enoxaparin Study Group. New Eng J Med 1999;341:793-800.

39. Leizorovicz A, Cohen AT, Turpie AG, et al. Randomised control trial of dalteparin for the prevention of venous thromboembolism in acutely ill medical patients. Circulation 2004;110:874-9.

40. Cohen AT, Davidson BL, Gallus AS, et al. Efficacy and safety offondaparinux for the prevention of venous thromboembolism in older acute medical patients: rando- 
mised placebo controlled trial. BMJ 2006;332:325-9.

41. Dentali F, Douketis JD, Gianni M, et al. Meta-analysis: anticoagulant prophylaxis to prevent symptomatic venous thromboembolism in hospitalized medical patients. Ann Intern Med 2007; 146:278-88.

42. Hull RD, Schellong SM, Tapson VF, et al. Extended-duration venous thromboembolism prophylaxis in acutely ill medical patients with recently reduced mobility: a randomized trial. Ann Intern Med 2010;153:8-18.

43. Goldhaber SZ, Leizorovicz A, Kakkar AK, et al. Apixaban versus enoxaparin for thromboprophylaxis inmedically ill patients. N Engl J Med 2011;365:2167-77.

44. Cohen AT, Spiro TE, Büller HR, et al. Rivaroxaban for thromboprophylaxis in acutely ill medical patients. $\mathrm{N}$ Engl J Med. 2013;368:513-23.

45. Kahn SR, Lim W, Dunn AS, et al. Prevention of VTE in nonsurgical patients: antithrombotic therapy and prevention of thrombosis, 9th ed: American College of Chest Physicians Evidence-Based Clinical Practice Guidelines. Chest 2012;141:195-226.

46. Kucher N, Spirk D, Kalka C, et al. Clinical predictors of prophylaxis use prior to the onset of acute venous thromboembolism in hospitalized patients. SWIss Venous ThromboEmbolism Registry (SWIVTER). J Thromb Haemost 2008;6:2082-7.
47. Masotti L, Righini M, Vuilleumier N, et al. Prognostic stratification of acute pulmonary embolism: focus on clinical aspects, imaging, and biomarkers. Vasc Health Risk Manag 2009;5:567-75.

48. Jiménez D, Aujesky D, Moores L, et al. Simplification of the pulmonary embolism severity index for prognostication in patients with acute symptomatic pulmonary embolism. Arch Intern Med 2010;170:1383-9.

49. Becattini C, Casazza F, Forgione C, et al. Acute pulmonary embolism: external validation of an integrated risk stratification model. Chest 2013;144:1539-45.

50. Goldhaber SZ. Surgical pulmonary embolectomy: the resurrection of an almost discarded operation. Tex Heart Inst J 2013;40:5-8.

51. Vasco B, Villalba JC, Lopez-Jimenez L, et al. Venous thromboembolism in nonagenarians. Findings from the RIETE Registry. Thromb Haemost 2009;101:1112-8.

52. Masotti L, Lorenzini G, Landini G, et al. New oral anticoagulants for acute and long term treatment of haemodinamically stable acute pulmonary embolism. Global J Respir Care 2013 [In press].

53. Budaj-Fidecka A, Kurzyna M, Fijałkowska A, et al. Inhospital major bleeding predicts mortality in patients with pulmonary embolism: an analysis of ZATPOL Registry data. Int J Cardiol 2013;168:3543-9. 